\title{
Standardized xeno- and serum-free culture platform enables large-scale expansion of high-quality mesenchymal stem/stromal cells from perinatal and adult tissue sources
}

\author{
Van T. Hoang ${ }^{1, * * *}$, Quynh-Mai Trinh ${ }^{1, * *}$, Dam Thi Minh Phuong ${ }^{1,3, * *}$, Hue Thi Hong Bui ${ }^{1,3}$, \\ Le Minh Hang ${ }^{1,3}$, Nguyen Thi Hong Ngan ${ }^{1,3}$, Nguyen Thi Tuyet Anh ${ }^{1,3}$, Phung Yen Nhi ${ }^{1}$, \\ Trinh Thi Hong Nhung ${ }^{1,3}$, Ha Thi Lien ${ }^{3}$, Tu Dac Nguyen ${ }^{3}$, Liem Nguyen Thanh ${ }^{1,2}$, \\ Duc M. Hoang ${ }^{1, *}$ \\ ${ }^{1}$ Vinmec Research Institute of Stem Cell and Gene Technology, Vinmec Healthcare System, Hanoi, Vietnam \\ ${ }^{2}$ Vinmec HiTech Center, Vinmec Healthcare System, Hanoi, Vietnam \\ ${ }^{3}$ College of Health Science, VinUniversity, Hanoi, Vietnam
}

\section{A R T I C L E I N F O}

\section{Article History:}

Received 6 May 2020

Accepted 8 September 2020

\section{Key Words:}

adipose tissue

bone marrow

mesenchymal stem cell

mesenchymal stromal cell

xeno- and serum-free cell manufacturing

umbilical cord

\begin{abstract}
A B S T R A C T
Background aims: Mesenchymal stem/stromal cells (MSCs) are of interest for the treatment of graft-versushost disease, autoimmune diseases, osteoarthritis and neurological and cardiovascular diseases. Increasing numbers of clinical trials emphasize the need for standardized manufacturing of these cells. However, many challenges related to diverse isolation and expansion protocols and differences in cell tissue sources exist. As a result, the cell products used in numerous trials vary greatly in characteristics and potency.

Methods: The authors have established a standardized culture platform using xeno- and serum-free commercial media for expansion of MSCs derived from umbilical cord (UC), bone marrow and adipose-derived (AD) and examined their functional characteristics.

Results: MSCs from the tested sources stably expanded in vitro and retained their biomarker expression and normal karyotype at early and later passages and after cryopreservation. MSCs were capable of colony formation and successfully differentiated into osteogenic, adipogenic and chondrogenic lineages. Pilot expansion of UC-MSCs and AD-MSCs to clinical scale revealed that the cells met the required quality standard for therapeutic applications.

Conclusions: The authors' data suggest that xeno- and serum-free culture conditions are suitable for largescale expansion and enable comparative study of MSCs of different origins. This is of importance for therapeutic purposes, especially because of the numerous variations in pre-clinical and clinical protocols for MSCbased products.
\end{abstract}

(C) 2020 International Society for Cell \& Gene Therapy. Published by Elsevier Inc. All rights reserved.

\section{Introduction}

The unique bioactivities of mesenchymal stem/stromal cells (MSCs), such as immunomodulation, anti-inflammation, promotion of tissue regeneration and angiogenesis, make them valuable candidates for regenerative medicine applications. MSC products have been approved in different markets to treat children with refractory and/or acute graft-versus-host disease and Crohn-related

\footnotetext{
* Correspondence: Van T. Hoang, PhD and Duc M. Hoang, PhD, 458 Minh Khai, Hanoi 100000, Vietnam.

** These authors contributed equally to this work.

E-mail addresses: v.vanht8@vinmec.com, v.duchm3@vinmec.com (V.T. Hoang).
}

enterocutaneous fistular disease. Potential clinical applications of MSCs are expanding quickly. According to https://clinicaltrials.gov (filtered as previously described [1]), as of 2019, a total of 921 clinical trials have been conducted for MSCs, making MSCs the second most popular cell source for therapy following hematopoietic stem cells. In addition to graft-versus-host disease and other immune-related disorder applications, MSC therapies have shown promising benefits in neurological and cardiovascular diseases, orthopedic complications and tissue repair/wound healing.

Bone marrow (BM), umbilical cord (UC) and adipose tissue (AD) are the most frequently used sources of MSCs, accounting for more than $90 \%$ of registered trials [1,2]. BM remains the most popular 
source of MSCs for therapeutic use, but growing numbers of trials have been conducted for UC-MSCs and AD-MSCs [1]. Other sources include placenta, dental pulp, oral mucosa, amniotic fluid and menstrual blood. MSCs isolated from different sources, including BM, AD, UC, placenta and tonsil tissue, exhibit distinguishable gene expression patterns [3-6]. In line with the molecular signatures, MSCs from different origins show tissue-specific diversity. MSCs isolated from perinatal tissues proliferate faster than those from adult tissue [7-9]. Moreover, MSCs are distinguished in many biological processes, such as secretion of cytokines and growth factors, immunomodulatory and anti-inflammatory behavior and mitochondria transfer activity [9-13]. These processes are widely accepted to be essential for MSC actions in many diseases [2,14]. Thus, understanding MSC characteristics and their underlying mechanisms in relation to their origin is essential in the search for the most potent cell source for a specific disease target of interest.

Diversity of tissue origin is just one of the challenges in MSC research. Even when the cells come from a particular tissue, many other parameters (e.g., isolation protocol, culture medium, oxygen tension, two- or three-dimensional system, manual culture or bioreactor) lead to heterogeneity in the final product, which is still generally considered to be MSCs. Culture medium significantly impacts the transcriptome of BM-MSCs and the properties of several MSC types $[15,16]$. Numerous culture medium variants have been using in preclinical and clinical studies. MSCs can be expanded with or without serum and in a xeno-containing or xeno-free medium, which can be homemade or commercial [17]. Because of the numerous medium variants, each MSC product resulting from a specific manufacturing protocol is unique. This might explain why it is so difficult to verify the efficacy of MSCs for targeted diseases and to identify subgroups of beneficial patients despite the long-term effort and expanding trial numbers. Notably, these trials are mostly in early phase 1 and 2 and have limited participants. In this case, meta-analysis is of importance. However, the bias of published data limits the power of such studies. Thus, more standardized MSC culture conditions will help in obtaining more reliable data and achieving a better understanding of MSC biology.

This study addresses some of these issues. First, the authors optimized a common xeno- and serum-free culture platform for MSCs derived from $\mathrm{UC}, \mathrm{AD}$ and $\mathrm{BM}$, which are the most frequently used MSC sources in clinical trials. Next, the authors characterized the expanded MSCs in early and advanced passages as well as after cryopreservation. Finally, the authors validated the platform by mimicking clinical-scale expansion of MSC products for potential therapeutic use.

\section{Methods}

Patient samples

A cohort of BM ( $n=16), A D(n=14)$ and UC $(n=30)$ samples obtained from healthy donors were collected in Vinmec International Hospital in 2019 after patients signed in an informed consent form. The authors also included cryopreserved samples of BM $(n=6)$ and $\operatorname{AD}(n=8)$ that were obtained between 2017 and 2018 in our hospital with the agreement of patients in the form of written informed consent. Sample collection and data analysis were approved by the ethics committee of Vinmec Healthcare System and were carried out in accordance with the Declaration of Helsinki.

\section{Isolation and culture of MSCs}

UC samples were obtained in $0.9 \%$ sodium chloride (Bidiphar, Quy Nhon, Vietnam) and stored at $4^{\circ} \mathrm{C}$. MSCs were isolated using enzymatic digestion methods. Briefly, the cord was cut into small fragments and incubated in $500 \mathrm{U} / \mathrm{mL}$ collagenase (Gibco, USA) at $37^{\circ} \mathrm{C}$ for $2.5 \mathrm{~h}$ in a gentleMACS dissociator (Miltenyi, Germany). The cells were seeded in treated cell culture flasks (Nunc; Thermo Fisher Scientific, USA).

Approximately $5 \mathrm{~g}$ of $\mathrm{AD}$ samples were collected in $0.9 \%$ sodium chloride (Bidiphar, Quy Nhon, Vietnam). The tissue was cut into small pieces and digested in $200 \mathrm{U} / \mathrm{mL}$ collagenase (Gibco, USA) for $1 \mathrm{~h}$ at $37^{\circ} \mathrm{C}$ in an Adi Plus multi-purpose medical centrifuge-VS-6030 (Vision Scientific Co, Ltd, Korea). Isolated cells were collected by centrifugation and plated in treated cell culture flasks (Nunc; Thermo Fisher Scientific, USA).

$\mathrm{BM}$ aspirates $(40 \mathrm{~mL})$ mixed with heparin were collected. Mononuclear cells were isolated using density-gradient centrifugation with Ficoll-Paque PREMIUM solution (GE Healthcare Life Sciences, USA). Harvested cells were seeded in treated cell culture flasks (Nunc; Thermo Fisher Scientific, USA).

Primary cells were cultured in MSC culture medium supplemented with $50 \mathrm{U} / \mathrm{mL}$ penicillin/streptomycin (Life Technologies, USA) at $37^{\circ} \mathrm{C}$ with $5 \%$ carbon dioxide. After 7 days, antibiotics were removed from the culture. MSCs were harvested when the cells reached $80 \%$ confluency. For long-term storage, MSCs were cryopreserved at passage (P) 0 and 1 in the serum-free, xeno-free and defined reagent CryoStor CS10 (STEMCELL Technologies, Canada) in the gas phase of liquid nitrogen in an automated Brooks system (Brooks Life Sciences, USA). The temperature was monitored and maintained at $-196^{\circ} \mathrm{C}$.

The authors performed a medium test for UC-MSCs employing commercial xeno- and serum-free media; namely, NutriStem MSC XF medium (Biological Industries, Israel), StemPro MSC serum-free medium (Gibco, USA), StemMACS MSC expansion media kit XF (Miltenyi, Germany), PowerStem MSC1 medium (PAN-Biotech, Germany) and MesenCult-ACF plus medium (STEMCELL Technologies, Canada). For $\mathrm{BM}$ and $\mathrm{AD}$, the last four MSC expansion media were tested. The primary cells were cultured in the media of interest directly after isolation to maintain the same in vitro conditions for the cells.

\section{Examination of population doubling time}

Growth curves were constructed for UC-, BM- and AD-MSCs from P2 to P6. For each passage, MSCs were seeded at a concentration of 5000 cells $/ \mathrm{cm}^{2}$ in triplicate in treated cell culture flasks (Nunc; Thermo Fisher Scientific) coated with the supplied attachment substrate for the MesenCult-ACF plus medium (STEMCELL Technologies, Canada) or with CELLstart coating substrate (Thermo Fisher Scientific, USA) for the other media and grown until the culture reached $80 \%$ confluency. The cells were harvested using TrypLE Select CTS enzyme (Gibco, USA). Cells were counted using a Neubauer improved C-chip disposable hematocytometer (INCYTO, Germany). Dead cells were stained with Trypan Blue. Population doubling time was calculated as described in the supplementary material. Each culture was performed in triplicate.

\section{Flow cytometry analysis}

MSC identity was examined by evaluating the expression of surface markers, specifically the positive markers CD73, CD90 and CD105 and the negative markers CD34, CD45, CD11b, CD19 and HLA-DR, using a human MSC analysis kit (Becton, Dickinson and Company, USA) and Navios (Beckmann Coulter, USA) and BD FACSCanto II (Becton, Dickinson and Company, USA) flow cytometers. Data analysis was performed using the Navios system and FlowJo software. The flow cytometry analysis was performed with fresh MSCs at P3 and P6 and cryopreserved MSCs after thawing at P3.

\section{Colony-forming unit assay}

UC-, AD- and BM-MSCs were cultured until P2 and were plated in triplicate at concentrations of 4,20 and 100 cells $/ \mathrm{cm}^{2}$, respectively. 
Cells were cultured in MSC expansion medium as described earlier, with medium exchange twice per week. After 14 days, cells were fixed with methanol and stained with Giemsa (Merck, Germany). Colony numbers were counted, and the morphology of colonies was observed under a microscope.

\section{Osteogenic, adipogenic and chondrogenic lineage differentiation assays}

The differentiation ability of isolated MSC samples was tested using a StemPro osteogenesis, adipogenesis and chondrogenesis differentiation kit (Gibco, USA) according to the manufacturer's instructions. The cells were cultured in the differentiation media for 14 days and then fixed with $4 \%$ paraformaldehyde (Sigma-Aldrich, USA). Differentiation of MSCs into osteogenic, adipogenic and chondrogenic lineages was detected using Alizarin Red S, Oil Red O and Alcian Blue (Sigma-Aldrich, Singapore), respectively. For details, see supplementary material.

\section{Immunomodulation assay}

To analyze the immunomodulatory capacity of MSCs, the authors performed co-culture assays between peripheral blood mononuclear cells (PBMCs) and MSCs. PBMCs were stained with carboxyfluorescein succinimidyl ester (CFSE) (Invitrogen, USA) and activated using phytohemagglutinin (PHA) (Life Technologies, USA). After 4 days of co-culture, the cells were labeled with anti-CD3, anti-CD4 and anti-CD8 antibodies (clones BW264/56, VIT4 and REA734, respectively; Miltenyi) and 7-aminoactinomycin D (Miltenyi, Germany) and then analyzed with a BD FACSCanto II flow cytometer (Becton, Dickinson and Company, USA). Non-activated PBMCs served as the negative control, and PHA-activated PBMCs without MSCs were the positive control. For details, see supplementary material.

\section{Karyotyping}

MSCs were cultured until P3 and P6 for karyotyping. To test genome stability after cryopreservation, MSCs were thawed and used for karyotype analysis at P3. Cells in metaphase were arrested using KaryoMAX Colcemid solution (Life Technologies, USA). Cells were incubated in $0.56 \%$ potassium chloride and fixed with Carnoy's fixative before being applied on slides. The samples were heated at $60^{\circ} \mathrm{C}$ overnight, treated with $0.05 \%$ trypsin (Gibco, USA) and then stained with Giemsa (Merck). Metaphases were analyzed using the metaphase system and Ikaros software (MetaSystems, Germany). For details, see supplementary material.

\section{Bacterial and fungal culture tests}

Cell supernatant was collected for bacterial and fungal culture tests. These were performed using the ISO 15189 certified microbiological laboratory of the diagnostic department at the Vinmec Times City International Hospital, Hanoi, Vietnam. Bacterial and fungal contamination was detected using the BacT/Alert three-dimensional microbial detection system (Biomerieux, USA). Mycoplasma was detected using a MycoAlert PLUS Mycoplasma detection kit (Lonza, Switzerland) and measured using a Lucetta luminometer (Lonza, Switzerland) following the manufacturer's instructions.

\section{Measurement of endotoxin}

Endotoxin measurement was performed using Food and Drug Administration-licensed Limulus amebocyte lysate (LAL) reagents and an Endosafe nexgen-PTS spectrophotometer (Charles River, USA) following the manufacturer's instructions. Briefly, the tested samples were diluted 10 times with LAL reagents, vortexed for $30-60$ seconds and inactivated at $80^{\circ} \mathrm{C}$ for $5 \mathrm{~min}$. After centrifugation at $3000 \mathrm{~g} / \mathrm{min}$ for $3 \mathrm{~min}, 25 \mu \mathrm{L}$ of sample was added into an Endosafe LAL cartridge and measured in the Endosafe nexgenPTS spectrophotometer.

\section{Statistical analysis}

If not otherwise indicated, data were analyzed using a two-sided Student's t-test with GraphPad Prism 8 software. Welch's correction was applied when significantly different standard deviations were observed. Analysis of variance (ANOVA) was performed to compare the means of more than two groups as indicated in the text. Statistical significance was defined as $P<0.05$.

\section{Results}

Identifying standard xeno- and serum-free culture conditions for expansion of $U C-, A D$ - and $B M-M S C S$

To standardize the culture conditions of all three MSC sources of interest, five commercially available media-namely, MesenCult-ACF plus medium (STEMCELL Technologies), NutriStem MSC XF medium (Biological Industries), PowerStem MSC1 medium (PAN-Biotech), StemMACS MSC expansion media kit XF (Miltenyi) and StemPro MSC serum-free medium (Gibco)-were tested for culture of newly isolated UC-MSCs ( $\mathrm{n}=4$, except $\mathrm{n}=2$ for the second medium). The cells were successfully expanded in StemMACS MSC expansion media kit XF (Miltenyi) and PowerStem MSC1 medium (PAN-Biotech), whereas the other media were unable to support the growth of the tested cell types. Therefore, the authors employed these two media for isolation and culture of UC-MSCs ( $\mathrm{n}=30$ and 9 , respectively). Comparison of the two media revealed that UC-MSCs cultured in StemMACS MSC expansion media kit XF (Miltenyi) required a longer time to reach confluence after isolation than those cultured in PowerStem MSC1 medium (PAN-Biotech) $(P=0.03)$ (see supplementary Figure $1 \mathrm{~A}$ ). Moreover, the cell numbers harvested in the StemMACS MSC expansion media kit XF (Miltenyi) at P0 and P1 were lower than the numbers harvested in the PowerStem MSC1 medium (PANBiotech) $(P=0.004$ and 0.006 , respectively) (Figure $1 \mathrm{~A})$.

Similarly, both StemMACS MSC expansion media kit XF (Miltenyi) and PowerStem MSC1 medium (PAN-Biotech) strongly supported the generation and growth of AD-MSCs ( $\mathrm{n}=6$ and 19, respectively). No significant difference in recovery time was observed between the two tested media $(P=0.9)$ (see supplementary Figure 1B). However, there was an increase in cell numbers in the first two passages propagated in the PowerStem MSC1 medium (PAN-Biotech) compared with the StemMACS MSC expansion media kit XF (Miltenyi) $(\mathrm{P} 0, P=0.04, \mathrm{P} 1, P=0.003)$ (Figure 1B).

In terms of BM-derived cells, purified mononuclear cells were propagated in StemMACS MSC expansion media kit XF (Miltenyi), MesenCult-ACF plus medium (STEMCELL Technologies), StemPro MSC serum-free medium (Gibco) and PowerStem MSC1 medium (PAN-Biotech) for the enrichment of MSCs ( $n=10,22,8$ and 2 , respectively). The cells successfully proliferated in all of the tested media (see supplementary Figure 1C). Furthermore, the numbers of harvested cells at P1 favored StemMACS MSC expansion media kit XF (Miltenyi) and MesenCult-ACF plus medium (STEMCELL Technologies) more than StemPro MSC serum-free medium (Gibco) $(P=0.01$ and $<0.0001$, respectively) (Figure 1C).

Regardless of culture medium and tissue source, isolated MSCs derived from UC, AD and BM showed the characteristic fibroblastic, spindle-shaped morphology of MSCs (Figure 1D-F). Taken together, these results illustrate that MSCs from UC, AD and BM can be isolated and expanded under the same xeno- and serum-free conditions and that StemMACS MSC expansion media kit XF (Miltenyi) and 
Top

A

UC

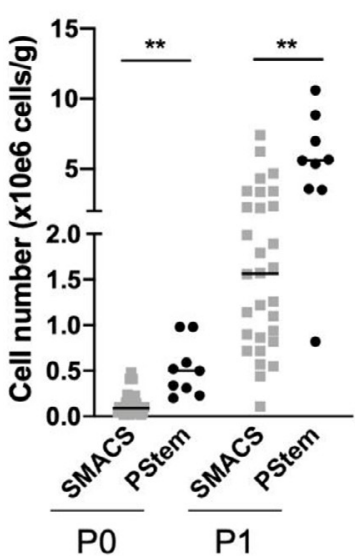

D UC

SMACS

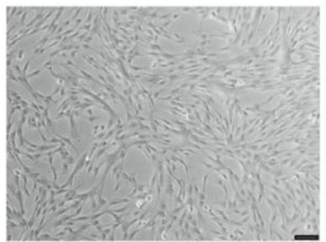

$\mathbf{F}$

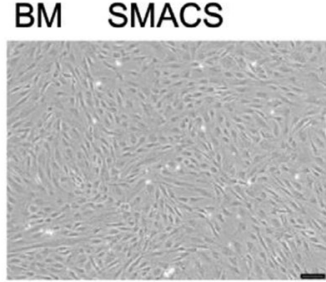

G
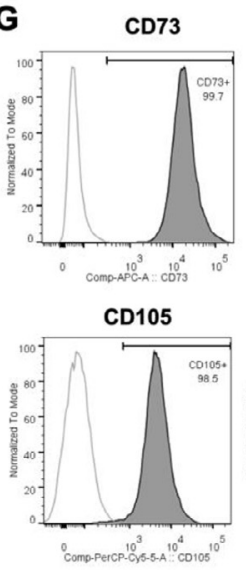

B

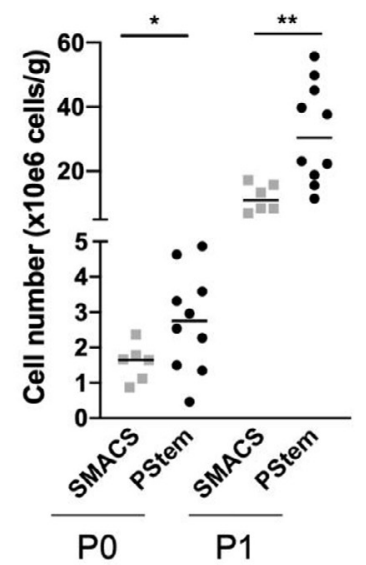

C

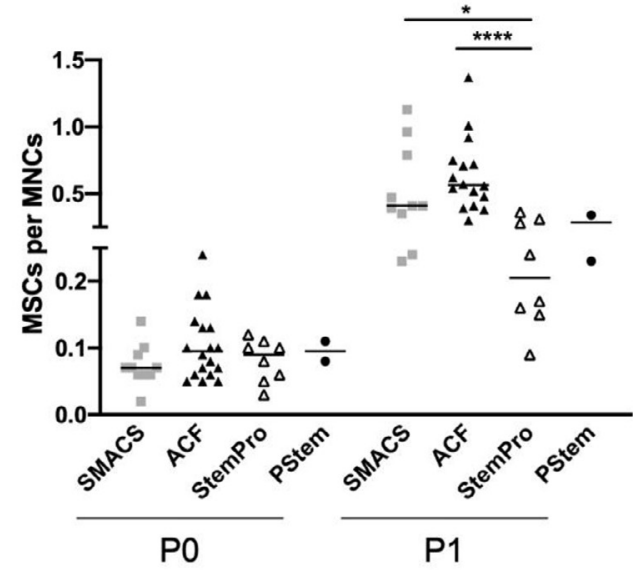

PStem

E $A D$

SMACS
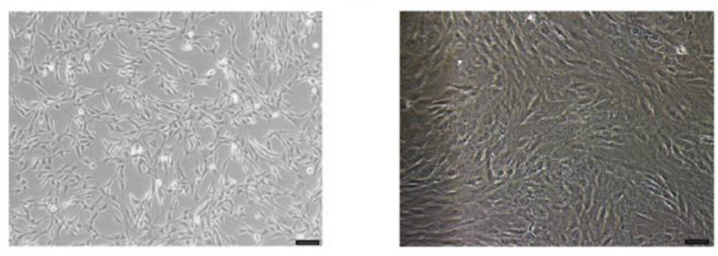

$\mathrm{ACF}$

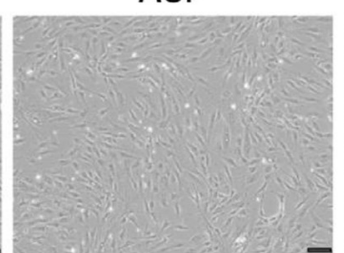

StemPro
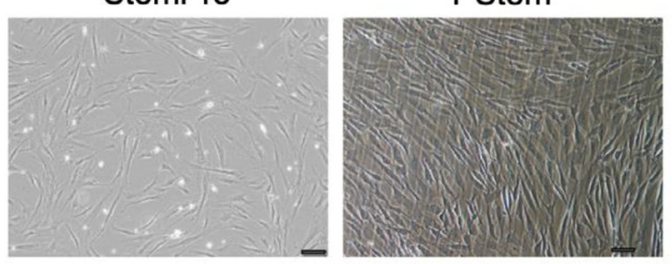

H
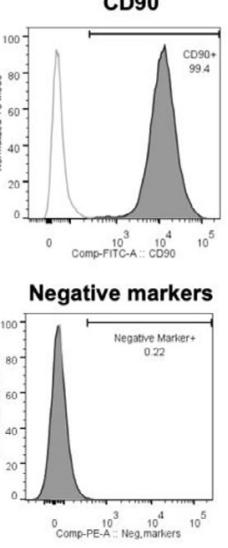

- CD73

- CD90

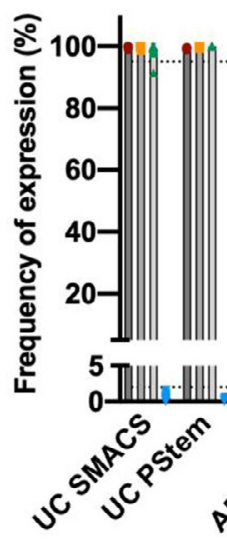

CD105

- Neg. markers

Figure 1. Isolation of MSCs from UC, AD and BM. (A) The number of UC-MSCs isolated after culture in SMACS was lower than that after culture in PStem medium ( $\mathrm{n}=30$ and 9 , respectively). (B) Similarly, AD-MSCs showed an increased yield at P0 and P1 in PStem compared with that seen in SMACS ( $n=10$ and 6 , respectively). (C) BM-MSC cell numbers harvested at P0 and P1 in SMACS, ACF, StemPro and PStem media demonstrate that SMACS and ACF were significantly more potent for isolation of MSCs than StemPro ( $\mathrm{n}=10,16,8$ and 2, respectively). (D-F) Morphology of isolated MSCs from UC (D), AD (E) and BM (F) in tested media, showing a spindle-shaped morphology of MSCs. (G) A representative example of BM-MSCs analyzed for MSC markers via flow cytometry, showing high expression of positive markers CD73, CD90 and CD105 and low expression of negative markers CD34, CD45, CD11b, CD19 and HLA-DR. (H) Expression analysis of MSC markers in UC-, AD- and BM-MSCs indicates a normal MSC phenotype in most of the tested cultures, except in AD- and BM-MSCs cultured in PStem, in which negative markers were upregulated. BM-MSCs cultured in PStem showed altered expression of CD90 and CD105. Dotted lines represent 95\% and $2 \%$, which are the cutoff values recommended by ISCT for positive and negative markers, respectively, in MSCs [18]. Scale bar, $50 \mu \mathrm{m} .{ }^{*} P<0.05,{ }^{* *} P<0.01,{ }^{* *} 0.001 \leq P<0.01$, ${ }^{* * * * *} P<0.0001$. ACF, MesenCult-ACF plus medium; MNCs, mononuclear cells; Neg., negative; PStem, PowerStem MSC1 medium; SMACS, StemMACS MSC expansion media kit XF; StemPro, StemPro MSC serum-free medium. (Color version of figure is available online). 
PowerStem MSC1 medium (PAN-Biotech) are potential medium candidates for a standardized culture platform.

\section{Flow cytometric characterization of MSCs under the tested xeno- and} serum-free conditions

To check MSC identity, the authors analyzed the surface marker expression of the isolated MSC samples using flow cytometry. Here the authors investigated the expression of the characteristic positive MSC markers CD73, CD90 and CD105 and the negative markers CD34, CD45, CD11b, CD19 and HLA-DR (Figure $1 \mathrm{G}$ ). The analysis revealed that the expression profiles of MSCs were dependent on the culture medium and tissue source (Figure $1 \mathrm{H}$; also see supplementary Table 1 ). Particularly, in UCMSCs cultured in StemMACS MSC expansion media kit XF (Miltenyi) and PowerStem MSC1 medium (PAN-Biotech), more than $95 \%$ of the cells were positive for CD73, CD90 and CD105, and less than $2 \%$ of the cells expressed the negative markers ( $\mathrm{n}=30$ and 8 , respectively). These expression levels met the recommendation of the International Society for Cell \& Gene Therapy (ISCT) for MSC identity evaluation [18].

By contrast, although AD-MSCs cultured in PowerStem MSC1 medium (PAN-Biotech) expressed high levels of positive markers, their expression of the negative markers was exaggerated, with a mean \pm standard deviation (SD) value of $8.67 \% \pm 12.80 \%(n=19)$. Of note, 11 of the 19 tested samples (57.89\%) showed more than $2 \%$ negative marker-expressing cells. By contrast, AD-MSCs in the StemMACS MSC expansion media kit XF (Miltenyi) culture exhibited the normal range of MSC markers recommended by the ISCT $(n=6)$. In the case of BM-MSCs, the PowerStem MSC1 medium (PAN-Biotech) induced negative marker expression in these cells $(45.8 \% \pm 54.0 \%)$, along with lower levels of CD90 (81.5\% $\pm 15.1 \%)$ and CD105 $(93.7 \% \pm$ $0.6 \%)(n=2)$ (Figure $1 \mathrm{H}$; also see supplementary Figure 1D). By contrast, the cells expanded in StemMACS MSC expansion media kit XF (Miltenyi), MesenCult-ACF plus medium (STEMCELL Technologies) and StemPro MSC serum-free medium (Gibco) displayed normal expression levels ( $\mathrm{n}=10,22$ and 8 , respectively) (Figure $1 \mathrm{H}$ ).

It is worth noting that biological variations might occur. In the authors' cohort, a UC-MSC sample in StemMACS MSC expansion media kit XF (Miltenyi) culture expressed a lower level of CD105, with $91.4 \%$ of cells positive, and a BM-MSC sample showed a slight increase in expression of negative markers, with $2.38 \%$ of cells positive, whereas the other analyzed markers remained within normal expression ranges.

Thus, although MSCs derived from various origins can be enriched in both StemMACS MSC expansion media kit XF (Miltenyi) and PowerStem MSC1 medium (PAN-Biotech), the flow cytometric analysis suggests that StemMACS MSC expansion media kit XF (Miltenyi) enabled the expansion of MSCs with consistent expression of surface markers.

\section{Characterization of cell growth and colony formation ability of MSCS} under the tested xeno- and serum-free conditions

To determine whether different culture media could potentially promote the proliferation of MSCs derived from various sources in the same manner, a growth curve analysis was performed for the cells from P2 to P6. As MSCs are recommended to be infused at early passages to avoid culture-induced genome instability, the analyzed passages were of interest for therapeutic applications. The results demonstrated that UC-MSCs cultured in StemMACS MSC expansion media kit XF (Miltenyi) proliferated faster than their counterparts cultured in PowerStem MSC1 medium (PAN-Biotech) $(P=0.006)$, with a mean \pm SD population doubling time (PDT) of $21.73 \mathrm{~h} \pm 3.43$ $\mathrm{h}$ versus $24.31 \mathrm{~h} \pm 1.77 \mathrm{~h}$ ( $\mathrm{n}=29$ and 8 , respectively) (Figure $2 \mathrm{~A}$ ). Furthermore, analysis of cell growth dynamics indicated that UC-MSCs cultured in PowerStem MSC1 medium (PAN-Biotech) showed reduced growth capacity at later passages, whereas growth remained more consistent under the StemMACS MSC expansion media kit XF (Miltenyi) conditions (one-way ANOVA, $P=0.006$ and 0.02 , respectively) (Figure 2B).

The trend was more prominent in the case of AD-MSCs. Although the cells were highly proliferative at the early passages in PowerStem MSC1 medium (PAN-Biotech), proliferation declined gradually at each subsequent passage (one-way ANOVA, $P<0.0001, \mathrm{n}=19$ ) (Figure 2D). By contrast, AD-MSCs cultured in StemMACS MSC expansion media kit XF (Miltenyi) behaved similar to UC-MSCs (one-way ANOVA, $P=0.038, n=7$ ) (Figure 2). Of note, there was no difference in the PDT of AD-MSCs expanded in these two media $(P=0.33)$ : the mean PDT was $35.67 \mathrm{~h} \pm 4.16 \mathrm{~h}$ in StemMACS MSC expansion media kit XF (Miltenyi) and $38.20 \mathrm{~h}$ $\pm 6.21 \mathrm{~h}$ in PowerStem MSC1 medium (PAN-Biotech) ( $\mathrm{n}=19$ and 7 , respectively) (Figure $2 \mathrm{C}$ ).

Similarly, the PDT of the BM-MSCs in StemMACS MSC expansion media kit XF (Miltenyi) and MesenCult-ACF plus medium (STEMCELL Technologies) was comparable (mean \pm SD, $38.44 \mathrm{~h} \pm 10.83 \mathrm{~h}$ and $37.98 \mathrm{~h} \pm 8.64 \mathrm{~h}, \mathrm{n}=9$ and 21 , respectively), whereas cells grown in StemPro MSC serum-free medium (Gibco) required significantly longer for population duplication $(120.20 \mathrm{~h} \pm 35.56 \mathrm{~h}, \mathrm{n}=8$ ) (Figure 2E). Similar to the other MSC types, StemMACS MSC expansion media kit XF (Miltenyi) maintained MSC growth over the analyzed time $(P=$ 0.14 ), whereas decreased cell division was observed for the cells cultured in MesenCult-ACF plus medium (STEMCELL Technologies) ( $P=$ 0.01 ) (Figure 2F). Of note, when comparing the PDT of MSCs isolated from the three tested sources, the authors noticed that UC-MSCs showed the highest proliferative properties, whereas adult-derived AD- and BM-MSCs divided significantly more slowly $(P<0.0001$ in both cases) (Figure 2G).

Additionally, observation of MSCs at P6 revealed that PowerStem MSC1 medium (PAN-Biotech) induced a change in AD-MSCs, which became round in shape, whereas AD-MSCs cultured in StemMACS MSC expansion media kit XF (Miltenyi) displayed the normal spindle-shaped morphology observed in earlier passages (Figure 2H). UCMSCs retained the characteristic MSC morphology under both conditions. However, the cells in PowerStem MSC1 medium (PAN-Biotech) required a longer time to reach confluence (Figure $2 \mathrm{H}$ ).

Colony formation ability of MSCs under the tested xeno- and serum-free conditions

In addition to growth curve analysis, the authors examined the colony formation ability of MSCs at P2 (Figure 3A). The colony-forming unit (CFU) numbers of UC-MSCs were similar in the StemMACS MSC expansion media kit XF (Miltenyi) and PowerStem MSC1 medium (PAN-Biotech) $(P=0.32, \mathrm{n}=29$ and 8 , respectively) (see supplementary Figure 1E), whereas AD-MSCs showed a higher colony formation potential in StemMACS MSC expansion media kit XF (Miltenyi) than in PowerStem MSC1 medium (PAN-Biotech) $(P=0.0011, \mathrm{n}=7$ and 12 , respectively) (see supplementary Figure $1 \mathrm{~F}$ ). The $\mathrm{CFU}$ numbers of BMMSCs tended to be higher in StemMACS MSC expansion media kit XF (Miltenyi) than in MesenCult-ACF plus medium (STEMCELL Technologies); however, the difference was not statistically significant $(P=0.13$, $\mathrm{n}=9$ and 19, respectively) (see supplementary Figure $1 \mathrm{G}$ ). The results also revealed that UC-MSCs had the highest CFU numbers $(P<0.0001)$, with 366 CFUs \pm 154 CFUs $(n=29)$, followed by AD-MSCs (97 CFUs \pm 18 CFUs, $\mathrm{n}=7$ ) and BM-MSCs, which had the lowest CFUs (16 CFUs \pm 3 CFUs, $n=9$ ) (Figure 3B).

Taken together, these data illustrate that although cells had longer recovery times and lower yields at the first two passages in StemMACS MSC expansion media kit XF (Miltenyi) than their counterparts in PowerStem MSC1 medium (PAN-Biotech), the cells expanded using the StemMACS MSC expansion media kit XF (Miltenyi) exhibited normal surface marker expression and high 
Top
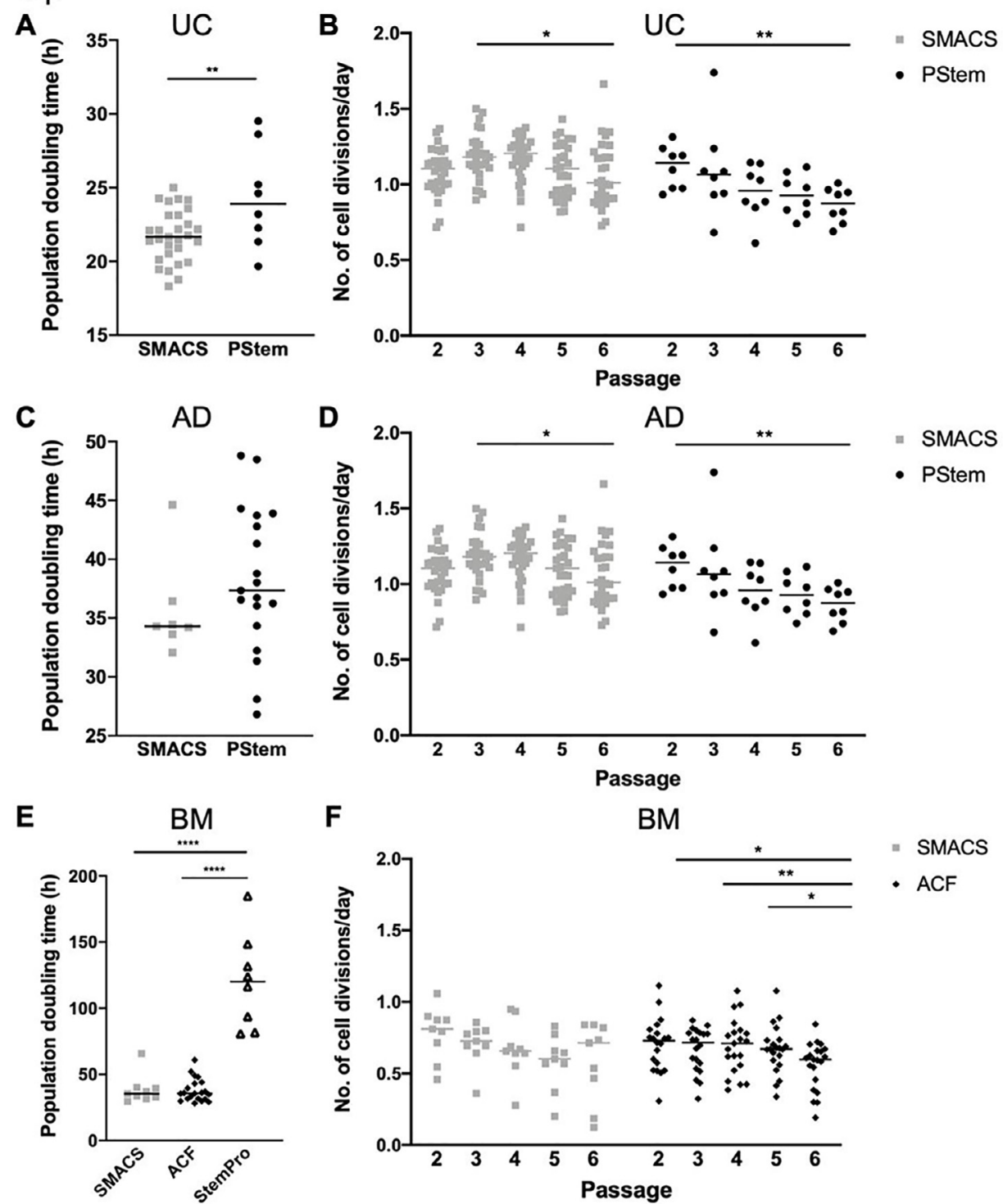

$\mathbf{F}$

BM

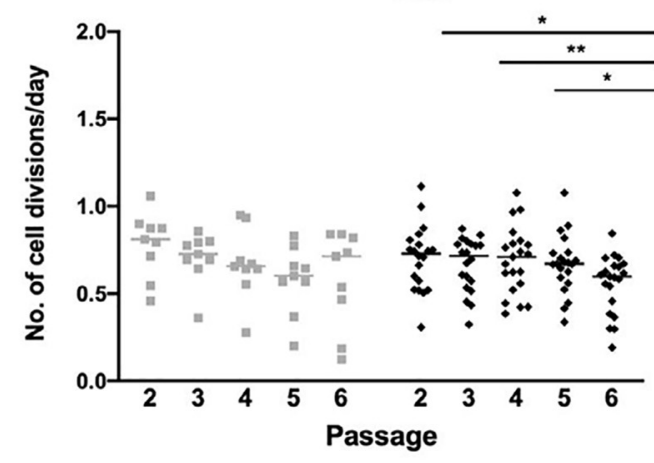

G

H
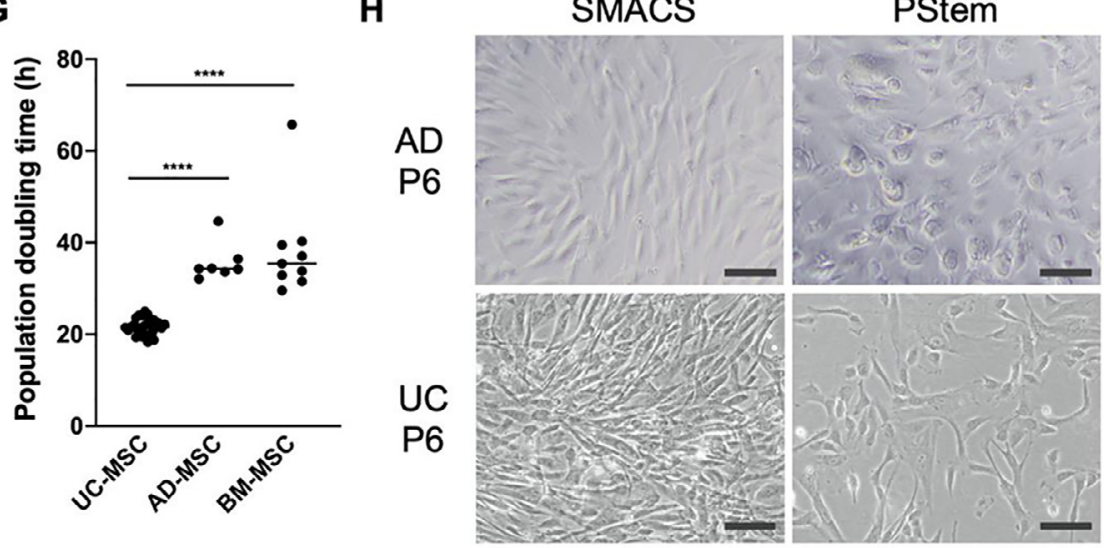

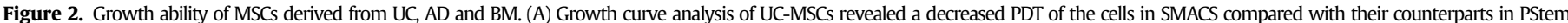

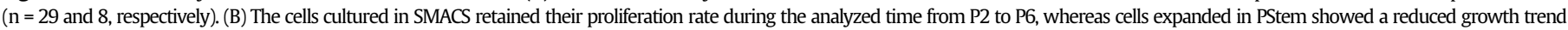

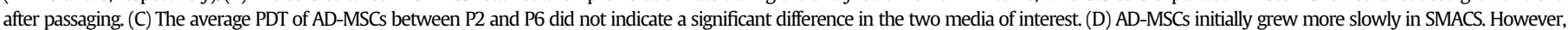

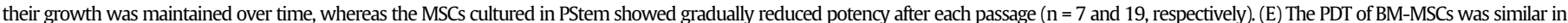

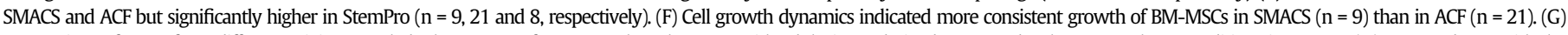

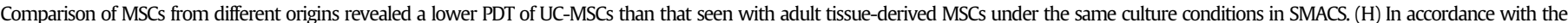

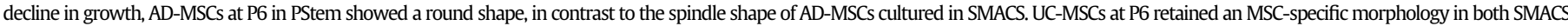

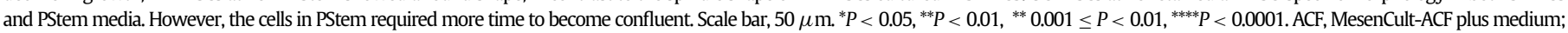
No., number; PStem, PowerStem MSC1 medium; SMACS, StemMACS MSC expansion media kit XF; StemPro, StemPro MSC serum-free medium. (Color version of figure is available online). 


\section{Top}

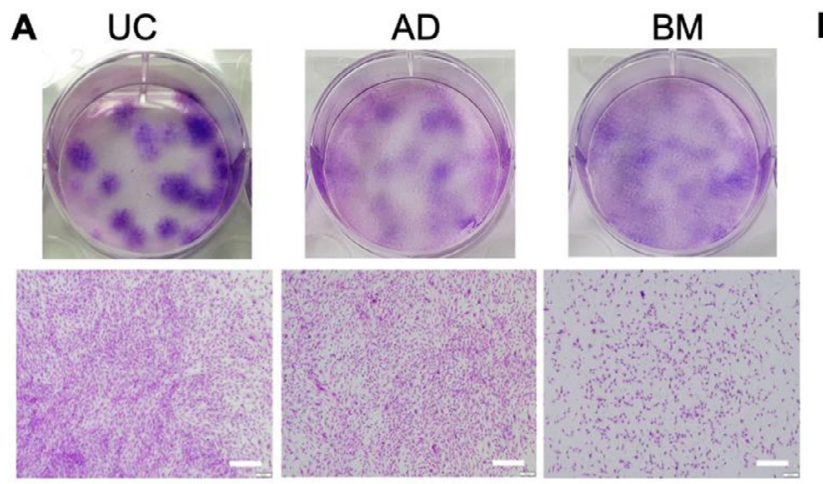

C

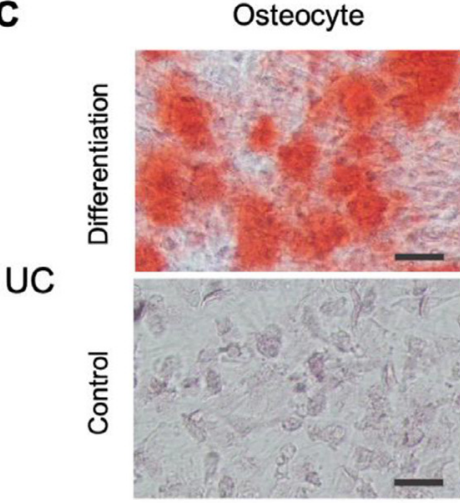

D

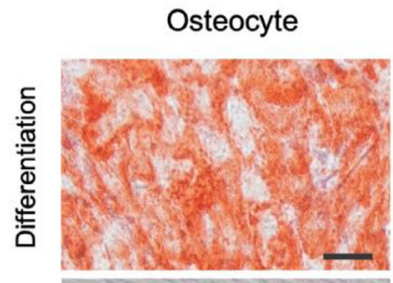

AD

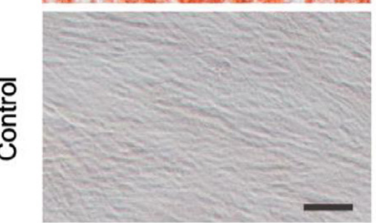

E

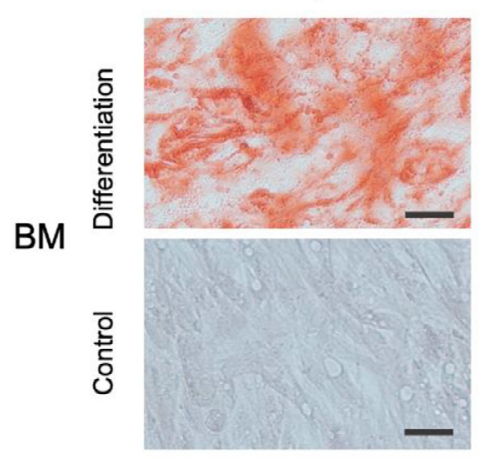

Adipocyte

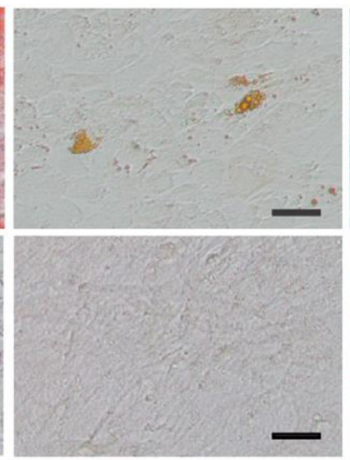

Adipocyte
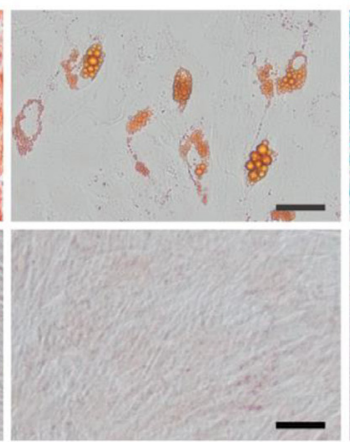

Adipocyte
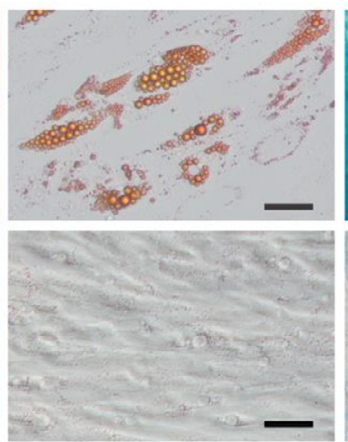

B

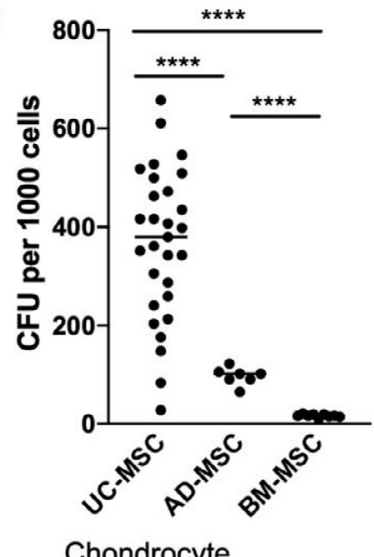

Chondrocyte

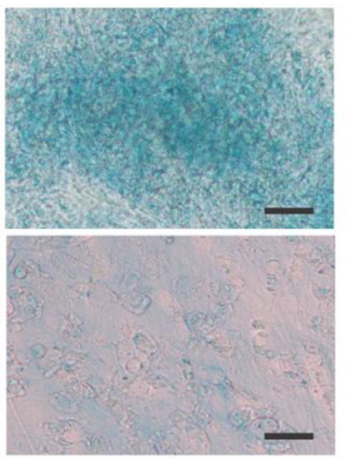

Chondrocyte
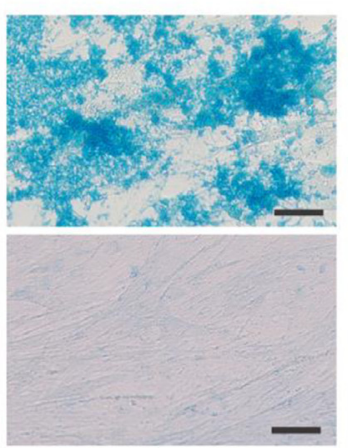

Chondrocyte
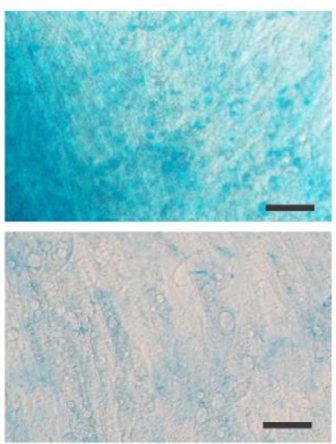

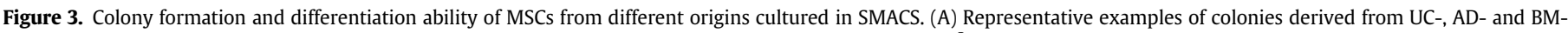

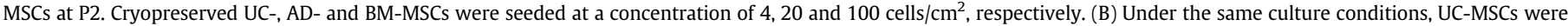

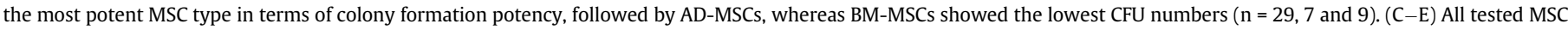

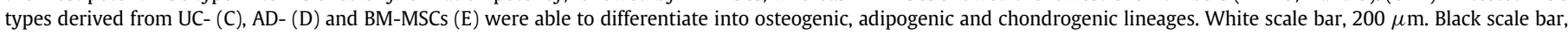
$50 \mu \mathrm{m}$. SMACS, StemMACS MSC expansion media kit XF. (Color version of figure is available online). 
Table 1

Differentiation of UC-, AD- and BM-MSCs into osteogenic, adipogenic and chondrogenic lineages.

\begin{tabular}{|c|c|c|c|c|c|c|}
\hline \multirow{2}{*}{$\begin{array}{l}\text { Medium } \\
\text { Results }\end{array}$} & \multicolumn{2}{|c|}{ SMACS } & \multicolumn{2}{|c|}{ PStem } & \multicolumn{2}{|c|}{ ACF } \\
\hline & Positive & Negative & Positive & Negative & Positive & Negative \\
\hline UC-MSCs & \multicolumn{2}{|c|}{$\mathrm{n}=29(\%)$} & \multicolumn{2}{|c|}{$\mathrm{n}=8(\%)$} & & \\
\hline Osteogenesis & $28(96.55)$ & $1(3.45)$ & $7(87.5)$ & $1(12.5)$ & & \\
\hline Adipogenesis & $29(100)$ & $0(0)$ & $8(100)$ & $0(0)$ & & \\
\hline Chondrogenesis & $29(100)$ & $0(0)$ & $8(100)$ & $0(0)$ & & \\
\hline AD-MSCs & \multicolumn{2}{|c|}{$\mathrm{n}=4(\%)$} & \multicolumn{2}{|c|}{$\mathrm{n}=14(\%)$} & & \\
\hline Osteogenesis & $4(100)$ & $0(0)$ & $14(100)$ & $0(0)$ & & \\
\hline Adipogenesis & $4(100)$ & $0(0)$ & $14(100)$ & $0(0)$ & & \\
\hline Chondrogenesis & $4(100)$ & $0(0)$ & $14(100)$ & $0(0)$ & & \\
\hline BM-MSCs & \multicolumn{2}{|c|}{$\mathrm{n}=10(\%)$} & & & \multicolumn{2}{|c|}{$\mathrm{n}=20(\%)$} \\
\hline Osteogenesis & $10(100)$ & $0(0)$ & & & $20(100)$ & $0(0)$ \\
\hline Adipogenesis & $10(100)$ & $0(0)$ & & & $20(100)$ & $0(0)$ \\
\hline Chondrogenesis & $10(100)$ & $0(0)$ & & & $20(100)$ & $0(0)$ \\
\hline
\end{tabular}

MSCs were cultured in SMACS, PStem and ACF media until P3, and expansion media were replaced with differentiation media for 14 days. The cells were fixed and stained with Alizarin S, Oil Red $O$ and Alcian Blue to observe osteogenic, adipogenic and chondrogenic differentiation, respectively. The number of analyzed samples and positive/negative tested samples and their frequencies are indicated in the table.

ACF, MesenCult-ACF plus medium; PStem, PowerStem MSC1 medium; SMACS, StemMACS MSC expansion media kit XF.

Table 2

Karyotype of UC-, AD- and BM-MSC samples.

\begin{tabular}{lllll}
\hline MSC type & Karyotype & P3 & P6 & P3 (cryo) \\
\hline \multirow{2}{*}{ UC-MSCs } & Normal karyotype (\%) & $28(93.34)$ & $27(90)$ & $29(96.67)$ \\
& Abnormality (\%) & $0(0)$ & $0(0)$ & $0(0)$ \\
& NA (\% of total) & $2(6.67)$ & $3(10)$ & $1(3.33)$ \\
AD-MSCs & Normal karyotype (\%) & $4(66.67)$ & $4(66.67)$ & $4(66.67)$ \\
& Abnormality (\%) & $0(0)$ & $0(0)$ & - \\
& NA (\% of total) & $2(33.33)$ & $2(33.33)$ & $2(33.33)$ \\
BM-MSCs & Normal karyotype (\%) & $6(60)$ & $7(70)$ & $8(80)$ \\
& Abnormality (\%) & $0(0)$ & $0(0)$ & $0(0)$ \\
& NA (\% of total) & $4(40)$ & $3(30)$ & $2(20)$ \\
\hline
\end{tabular}

Karyotyping was performed with fresh MSCs at P3 and P6 and with cryopreserved samples at P3 ( $n=30,6$ and 10 , respectively). Overall, the samples showed a normal karyotype. Of note, $\operatorname{inv}(9)(p 11 q 13)$ was observed in one AD-MSC sample. The pericentric inversion on chromosome 9 is a common population inversion variant and is not counted as a mutation. For each sample, 20 metaphases were counted, if not mentioned otherwise. In some samples, less than 12 metaphases were detected, and thus no results were available. These samples and samples that were not analyzed are marked as NA.

NA, not applicable.

colony formation capacity and maintained their long-term proliferative bioactivity, findings observed in all three main sources: UC, AD and BM.

Differentiation capacity of MSCs under the tested xeno- and serum-free conditions

To investigate the effects of different media on the differentiation capacity of AD-, BM- and UC-MSCs, tri-lineage differentiation assays were performed, and the results demonstrated that all the analyzed MSC types were able to differentiate into osteogenic, adipogenic and chondrogenic lineages (Figure $3 \mathrm{C}-\mathrm{E}$, Table 1 ). In comparison to AD- and BM-MSCs, UC-MSCs exhibited lower adipogenesis potential (see supplementary Figure 2A). Furthermore, UC-MSCs required a longer time for osteogenic differentiation (see supplementary Figure 2B).

\section{Maintaining quality of MSCs from different origins after} cryopreservation and prolonged in vitro cultivation

To assess the viability of the freshly harvested cells upon cryopreservation, MSC samples were subjected to a Trypan Blue assay
( $\mathrm{n}=30,4$ and 10 for UC-, AD- and BM-MSCs, respectively) (Figure 4A). Overall, the fresh cells at P1 showed high viability, with living cell frequencies (mean \pm SD) of $98.45 \% \pm 0.92 \%, 97.83 \% \pm$ $3.21 \%$ and $98.8 \% \pm 2.49 \%$ in UC-, AD- and BM-MSCs, respectively. The cryopreservation process might reduce the viability of cells; however, a reasonable survival rate was observed in all the analyzed samples (mean \pm SD), with $90.65 \% \pm 5.75 \%$ UC-MSCs, 96.22\% $\pm 4.36 \%$ ADMSCs and $90.33 \% \pm 7.62 \%$ BM-MSCs. Additionally, prolonged in vitro expansion until P6 did not affect cell survival compared with the younger P1 counterparts (see supplementary Figure 3A).

Furthermore, the authors observed a normal marker profile in cryopreserved MSCs and P6 cells, in which more than 95\% of the cells expressed CD73, CD90 and CD105 and as low as 2\% of the cells showed negative expression of the CD34, CD45, CD11b, CD19 and HLA-DR markers (Figure 4B; also see supplementary Figure 3B).

Next, the authors analyzed the influence of cryopreservation on cell growth and colony formation ability of MSCs. For this purpose, the authors divided fresh cells into two parts. The first part was further expanded to calculate growth ability and used for the CFU assay at P2. The other part was cryopreserved. After 1 month, cells were thawed and tested for cell growth and CFU capacity at the same passage. The cell growth ability of UC- and AD-MSCs was comparable between the fresh and cryopreserved/thawed samples, but thawed BM-MSCs grew more slowly than the fresh cells (Figure 4C). In addition, cryopreservation did not alter the CFU numbers of UC- and ADMSCs (Figure 4D,E). CFU data of fresh BM were not available in this study. The authors also tested the influence of prolonged in vitro culture on colony formation, which showed that UC- and BM-MSCs maintained their potential at P6 (CFU of P2 compared with P6, $P=0.2$ and $P=0.75$, respectively) (see supplementary Figure 3C,E). AD-MSCs at P6 showed lower CFU numbers than their counterparts at P2 $(P=0.01)$ (see supplementary Figure 3D).

To evaluate the genetic stability of MSCs cultured in StemMACS MSC expansion media kit XF (Miltenyi), the authors performed karyotype analysis at $\mathrm{P} 3$ and $\mathrm{P} 6$ of both freshly cultured and cryopreserved samples (UC, $A D$ and $B M, n=30,6$ and 10 , respectively) Table 2. The results showed that all tested samples maintained the normal karyotypes (Figure $4 \mathrm{~F}-\mathrm{H}$ ). In total, 117 karyograms of over 133 tested samples (88\%) met the analysis standard, with no mutations or major alterations in chromosome number and structure (see supplementary Table 2). One AD-MSC sample showed pericentric inversion on chromosome 9-inv(9)(p11q13), which is a common population inversion variant-at both P3 and P6. In 16 of 133 samples 


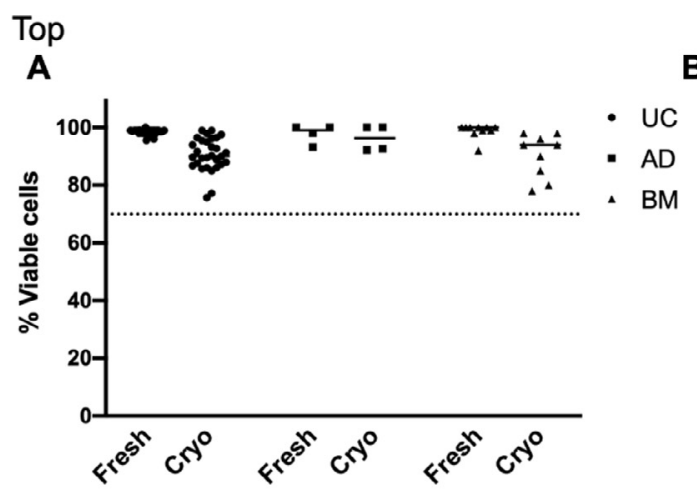

C
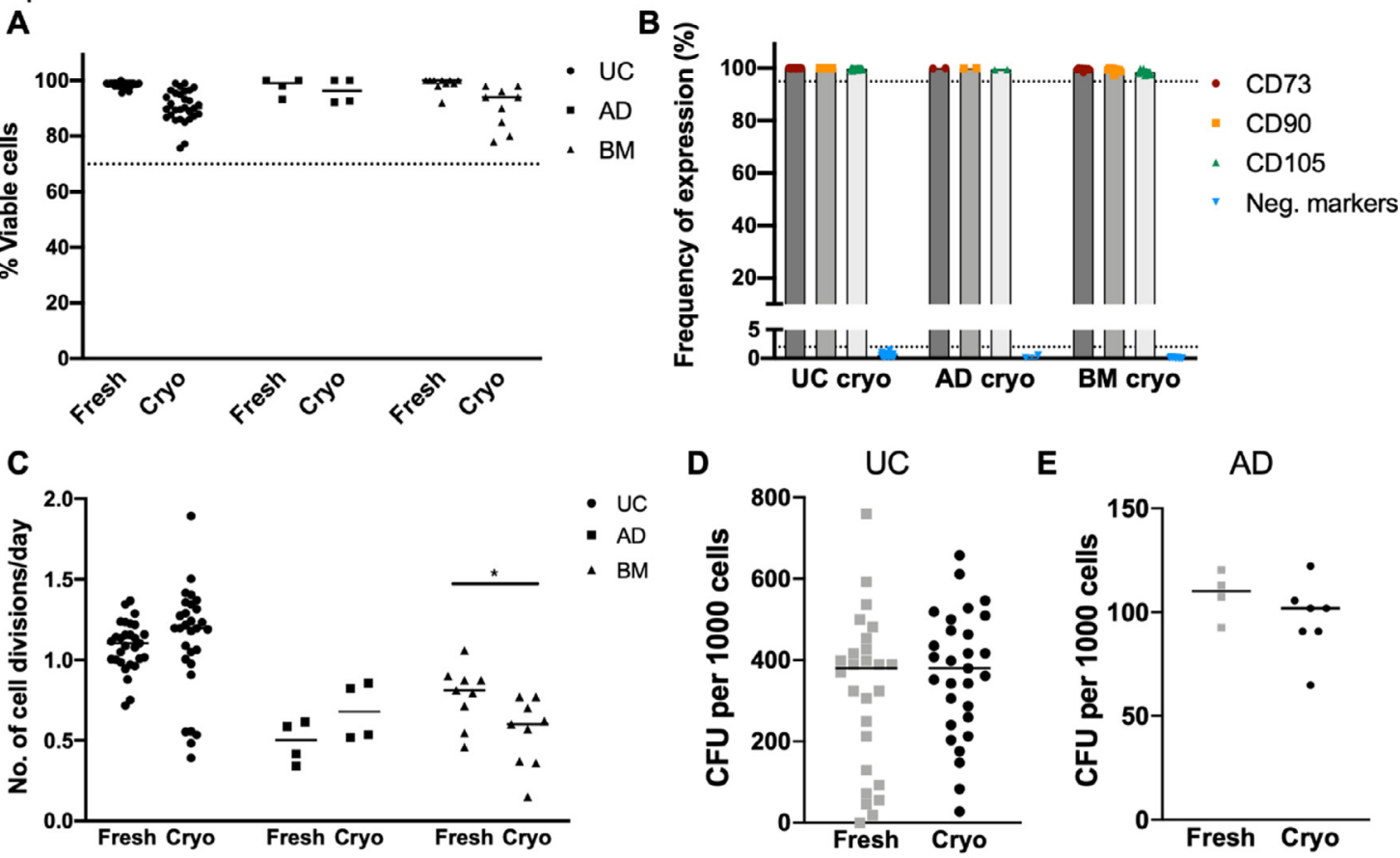

E

$A D$

$\mathbf{F}$

G

$A D$

H
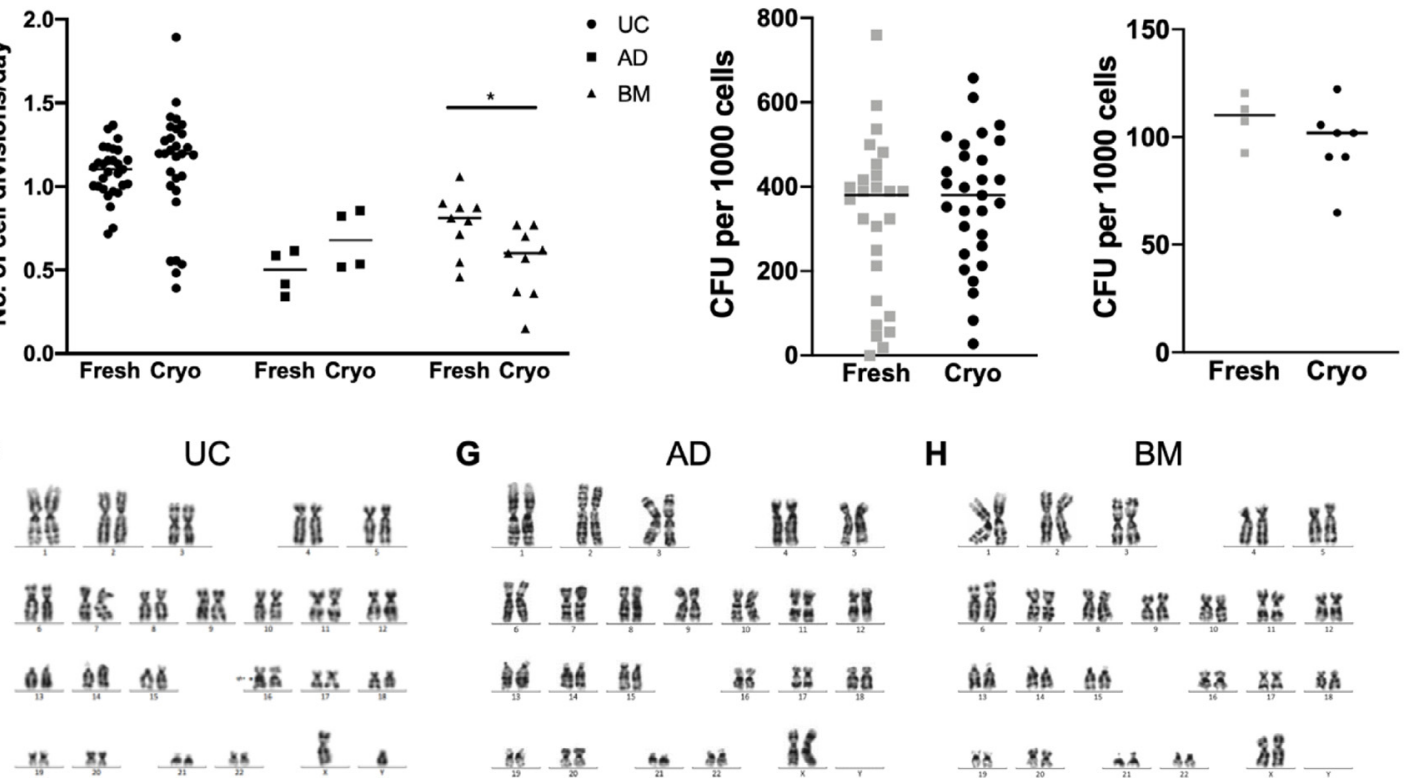

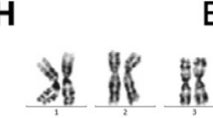

BM
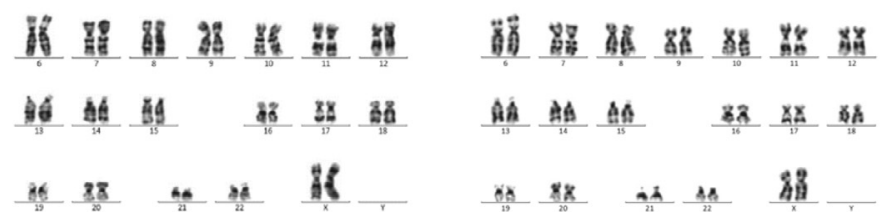

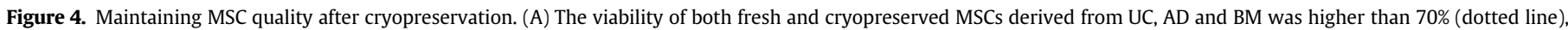

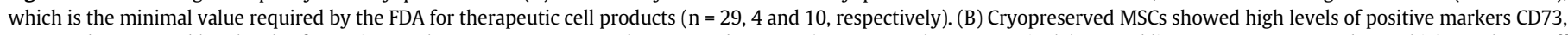

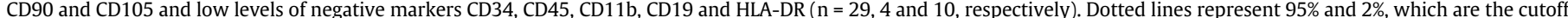

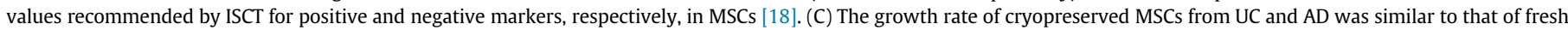

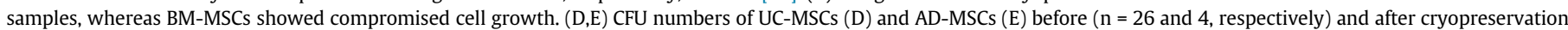

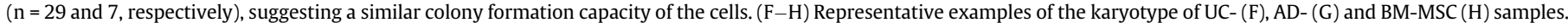
$0.01 \leq{ }^{*} \mathrm{P}<0.05$. Cryo, cryopreserved; FDA, Food and Drug Administration; Neg., negative; No., number. (Color version of figure is available online).

(12\%), the number of metaphases was too low for the analysis. Overall, the results indicated that the cultured MSCs showed a normal karyotype at P3 and P6 in fresh samples and after thawing of cryopreserved samples.

\section{Expansion of MSCs from UC and AD under optimized conditions}

To demonstrate that the authors' xeno- and serum-free platform might support large-scale production of MSCs, UC- and ADMSCs were expanded to clinically relevant numbers. For this purpose, one vial of two million cryopreserved UC-MSCs at P1 was thawed and manually cultured until P5 $(n=2)$ (Figure $5 \mathrm{~A})$. The results suggested that expansion of UC-MSCs as much as $5976 \pm$ 966 times was feasible within 14 days. Similarly, fresh AD-MSCs were tested for in vitro expansion capacity from P0 to P3 $(\mathrm{n}=3)$ (Figure 5B), and the cells were able to expand exponentially during the analyzed time.

MSC products must undergo stringent quality control tests before application in humans, as depicted in Figure 5C. Here the authors analyzed cell identity using flow cytometry and assessed the potency of differentiation and the immunomodulatory ability of UC-MSCs.
Furthermore, the authors recorded cell viability using Trypan Blue, sterility (bacteria, fungi, Mycoplasma), purity via endotoxin measurement and karyotype. All expanded MSC samples showed normal marker expression (Figure 5D). UC- and AD-MSCs were capable of multi-lineage differentiation: osteogenesis, adipogenesis and chondrogenesis (Figure 5E,F). A second tested potency assay was related to immunomodulation of UCMSCs. For this assay, PBMCs $(n=6)$ were activated with PHA and cultured with UC-MSCs. After 4 days, the authors analyzed frequencies of proliferating fractions, which included cells with low levels of carboxyfluorescein succinimidyl ester among the viable $\mathrm{CD} 3^{+} \mathrm{CD} 4^{+} \mathrm{T}$-helper cells and $\mathrm{CD}^{+} \mathrm{CD}^{+}$cytotoxic T cells (Figure 5G). Indeed, UC-MSCs significantly inhibited proliferation of both $\mathrm{CD}^{+} \mathrm{CD} 4^{+} \mathrm{CD} 8^{-} \mathrm{T}$-helper cells and $\mathrm{CD}^{+} \mathrm{CD}^{-} \mathrm{CD}^{+}$cytotoxic T cells (Figure $5 \mathrm{H}$ ). Similarly, AD-MSCs tended to reverse the activation of these T-cell subsets by PHA (see supplementary Figure 3F). Finally, the harvested cells showed high viability and no contamination with bacteria, fungi or Mycoplasma, and endotoxin levels were under the detection limit, which was $0.05 \mathrm{EU} / \mathrm{mL}$. Karyotype analysis confirmed a normal karyotype (Figure 5I). Overall, the authors' optimized culture platform enabled a clinically relevant scale expansion of MSCs that met all critical quality control requirements of therapeutic cellular products. 


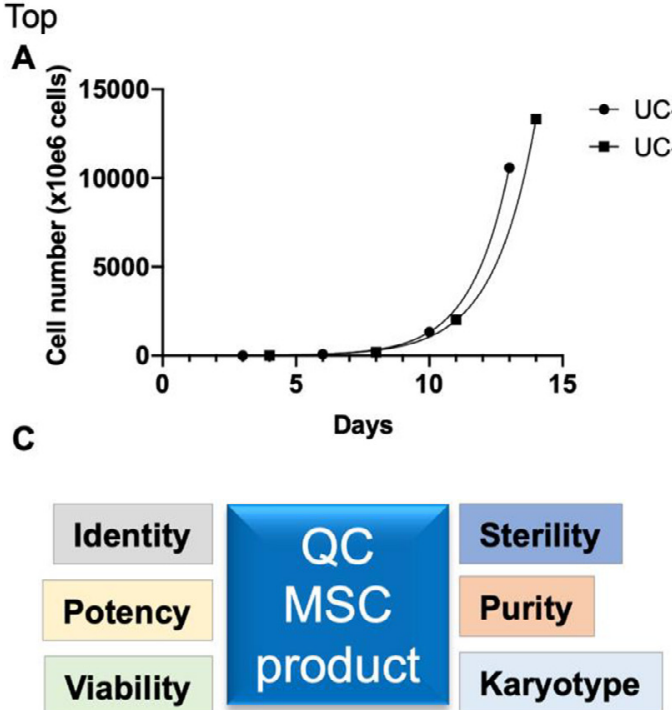

E

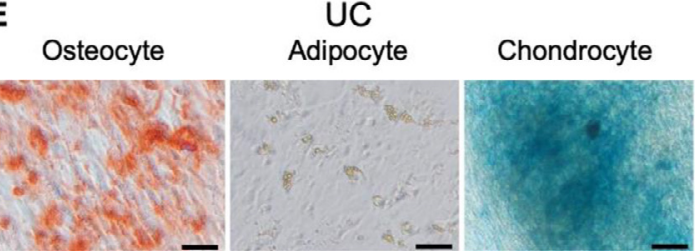

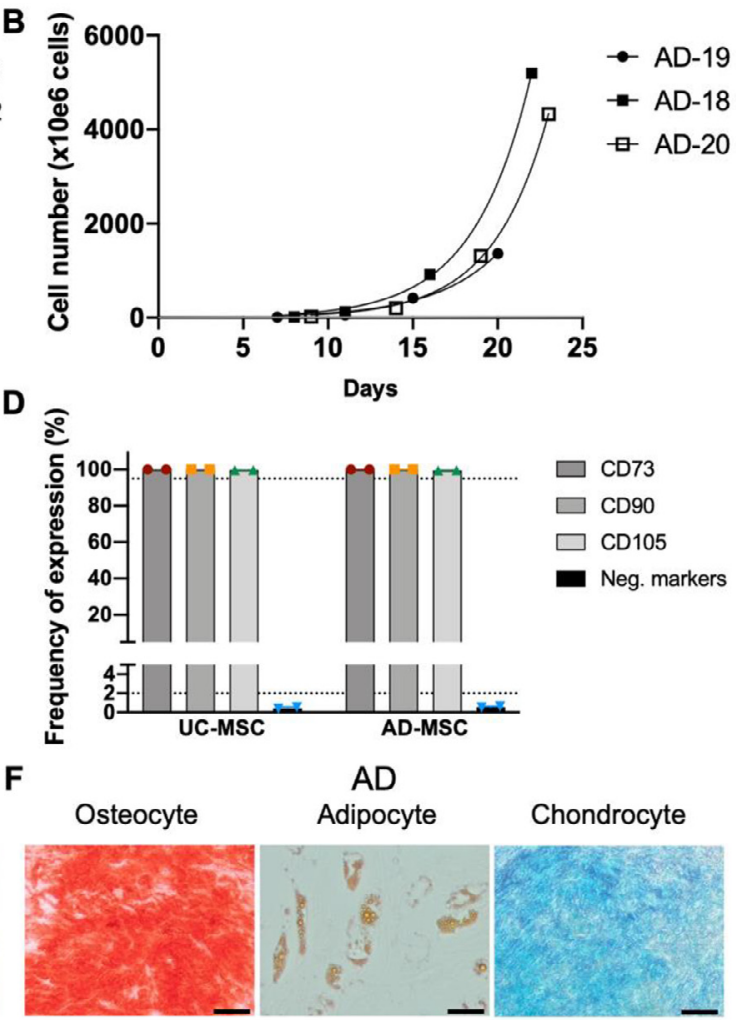

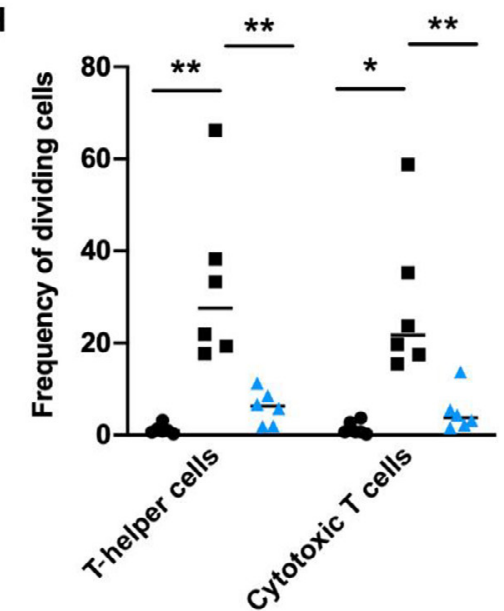

H
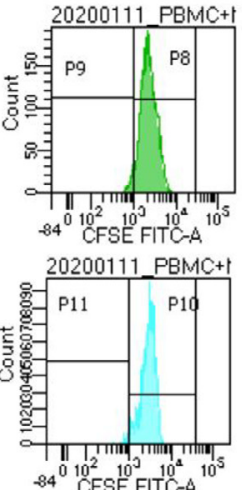

$84^{4}{ }^{10^{2}}{ }^{10^{3}}{ }^{10^{4}}{ }^{10}$

I

G
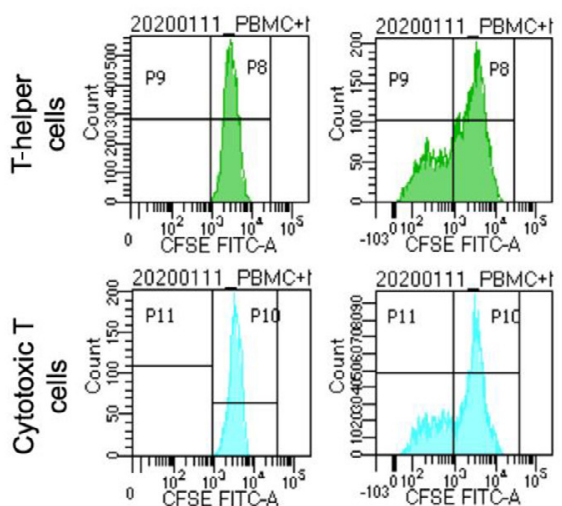


\section{Discussion}

Because of the regenerative and immunoregulatory features of MSCs derived from either perinatal or adult tissues, MSCs are a valuable resource for clinical trials and regenerative medicine [2]. The majority of published culture conditions for MSCs in both research and clinical settings utilize fetal bovine serum (FBS) or human platelet-derived supplement as the main source of growth factors to support MSC proliferation and stemness [17]. However, their limitations, which include batch-to-batch variation, non-defined components and potential side effects due to contamination with micro-organisms and endotoxin, have been recently addressed [19-22]. Moreover, human BM- and AD-MSCs are better able to expand in a xenoand serum-free medium than in FBS-containing culture [23]. Here, because of the current need for more standardized and safer MSC therapeutic products, the authors established a xeno- and serum-free culture protocol for MSCs derived from UC, AD and BM. Xeno- and serum-free culture conditions have been investigated in previous studies [16,24,27]. Most of these studies compared MSC properties under xeno- and serum-free conditions with those of their counterparts cultured in the presence of FBS [24-27] and/or human platelet lysates [21], and the results support potential replacement of xenogenic and serum-derived components in the MSC culture, especially for clinical applications. A recent study also tested a newly developed xeno- and serum-free medium for UC-, AD- and BM-MSCs, which were obtained from third-party suppliers [16]. Massive clonal selection of MSCs over time, especially at early passages, has been reported [28]. This suggests the fundamental importance of standardized conditions for the initial culture to reduce bias and heterogeneity in subsequent observations. To the best of the authors' knowledge, we are the first to intensively evaluate commercial xenoand serum-free culture reagents and report a common platform for isolation and propagation of MSCs from all three of the mentioned tissue sources.

Comprehensive analysis of both fresh cultured cells and preserved cells grown in StemMACS MSC expansion media kit XF (Miltenyi), in combination with CELLstart coating substrate (Thermo Fisher Scientific) and TrypLE (Gibco), demonstrated a number of findings: (i) all tested AD-, BM- and UC-MSCs cultured under the authors' conditions exhibited basic MSC characteristics as defined by the ISCT [18]; (ii) cells maintained karyotype normality after six consecutive passages; (iii) UCMSCs had the highest proliferation rate compared with their AD and BM counterparts, and similar results were observed in the CFU assay; (iv) cryopreservation of these MSCs under xeno- and serum-free conditions did not alter cell quality or characteristics; and (v) the authors' platform competently supported clinical-scale production and quality control requirements according to the current guidelines [29,30].

Another challenge-but also a great opportunity-in the MSC research field is related to the large number of potential tissue donors. Although MSCs derived from adult sources, such as BM and AD, are commonly used [1], the invasiveness of the procedures required for obtaining these tissues, the risk of complications and the age-dependent reduction in cell quality have become potential obstacles in MSC application [31-33]. Recently, UC-MSCs-a perinatal MSC sourcehave emerged as an alternative cell source, allowing the development of allogeneic transplantation for various diseases because of several advantages, such as ease of obtainment, non-invasive retrieval method and absence of ethical barriers. Although differently originated MSCs show some similarities, such as morphology, marker identity and multi-lineage differentiation capacity, they largely differ in many other bioactivities (e.g., growth ability, differentiation into certain lineages, anti-inflammatory and immunomodulatory capacity and genetic and epigenetic signature) $[8,9,12,34-36]$. In this study, the authors have shown that UC-MSCs exhibit more potent in vitro expansion and CFU formation than their adult counterparts. Although MSCs from all analyzed sources were capable of differentiation, UC-MSCs showed compromised adipogenic and osteogenic differentiation compared with AD- and BM-MSCs. These results confirm previous findings showing that MSC characteristics depend on tissue origin. Further comparative study of MSCs from different tissues is of interest to identify the best and most potent MSC source for each disease entity. The authors' standardized platform is critical for this approach, helping MSC applications be more precise and targeted.

Since 2018, allogeneic AD-MSCs have been authorized in the European Union for treatment of complex perianal fistulas in Crohn disease [37,38]. As allogeneic MSCs are increasingly used, Good Manufacturing Practice (GMP)-compliant, large-scale production has been of interest. By using a commercial medium, the authors might reduce bias between MSC manufacturing centers. The StemMACS MSC expansion media kit XF (Miltenyi) is not a GMP product. However, the related GMP product, MSC-Brew GMP medium (Miltenyi), is based on the formulation of the StemMACS MSC expansion media kit $\mathrm{XF}$ (Miltenyi), and thus translation of this product into GMP manufacturing for clinical applications would be feasible [39].

\section{Conclusions}

In sum, the authors have developed a standardized platform for xeno- and serum-free culture of MSCs from different origins. The authors demonstrated that the quality of expanded MSCs was conserved after in vitro culture and cryopreservation. Moreover, the standardized culture platform enables further comparative studies to understand the biology of MSCs depending on their origin. These studies would be beneficial for application of MSCs in a clinical setting and for investigation of the underlying mechanisms.

\section{Funding}

This work was funded by a Vingroup research grant (project nos. PRO.19.48 and PRO.19.49).

\section{Declaration of Competing Interest}

The authors of the study are employed by the not-for-profit Vinmec Healthcare System.

\section{Author Contributions}

Conception and design of the study: DMH, LTN, VTH, DTMP and QMT. Acquisition of data: DMH, VTH, DTMP, QMT, HTHB, LMH, NTHN, NTTA, PYN, TTHN, HTL, TDN and LNT. Analysis and interpretation of data: VTH, DMH, DTMP and QMT. Drafting or revising the manuscript: VTH, DMH, DTMP, QMT, LTN, HTHB, LMH, NTHN, NTTA, PYN, TTHN, HTL and TDN. All authors have approved the final article.

\section{Acknowledgments}

The authors thank our collaborating clinicians at the Vinmec Healthcare System for collecting samples for this study and the microbiology unit of the laboratory department at the Vinmec Times City International Hospital for performing micro-organism tests. The authors thank Bui Viet Anh, MSc, and Associate Professor Tran Thi Thanh Huong, MD, and their colleagues at the HiTech Center, Vinmec Healthcare System, for their support with the quality control analysis. The authors appreciate the scientific input and support of the Vinmec Scientific Committee and the Vinmec Ethics Committee. Finally, the authors' special gratitude goes to all volunteers who donated primary materials for the research. The manuscript was edited by AJE under certificate number 4037-A8A0-CBD9-A7F1-E375. 


\section{Supplementary materials}

Supplementary material associated with this article can be found in the online version at doi:10.1016/j.jcyt.2020.09.004.

\section{References}

[1] Kabat M, Bobkov I, Kumar S, Grumet M. Trends in mesenchymal stem cell clinical trials 2004-2018: is efficacy optimal in a narrow dose range? STEM CELLS Transl Med 2020;9:17-27.

[2] Pittenger MF, Discher DE, Péault BM, Phinney DG, Hare JM, Caplan AI. Mesenchymal stem cell perspective: cell biology to clinical progress. Npj Regen Med 2019;4:22.

[3] Abu Kasim NH, Govindasamy V, Gnanasegaran N, Musa S, Pradeep PJ, Srijaya TC et al. Unique molecular signatures influencing the biological function and fate of post-natal stem cells isolated from different sources: gene expression of mesenchymal stem cells from various sources. J Tissue Eng Regen Med 2015;9:E252-66.

[4] Cho K-A, Park M, Kim Y-H, Woo S-Y, Ryu K-H. RNA sequencing reveals a transcriptomic portrait of human mesenchymal stem cells from bone marrow, adipose tissue, and palatine tonsils. Sci Rep 2017;7:17114.

[5] Covas DT, Panepucci RA, Fontes AM, Silva WA, Orellana MD, Freitas MCC, et al. Multipotent mesenchymal stromal cells obtained from diverse human tissues share functional properties and gene-expression profile with CD146+ perivascular cells and fibroblasts. Exp Hematol 2008;36:642-54.

[6] Roson-Burgo B, Sanchez-Guijo F, Del Cañizo C, De Las Rivas J. Insights into the human mesenchymal stromal/stem cell identity through integrative transcriptomic profiling. BMC Genomics 2016;17:944.

[7] Bakopoulou A Apatzidou D, Aggelidou E, Gousopoulou E, Leyhausen G, Volk J, et al. Isolation and prolonged expansion of oral mesenchymal stem cells under clinical-grade, GMP-compliant conditions differentially affects "stemness" properties. Stem Cell Res Ther 2017:8:247.

[8] Kern S, Eichler H, Stoeve J, Klüter H, Bieback K. Comparative Analysis of Mesenchymal Stem Cells from Bone Marrow, Umbilical Cord Blood, or Adipose Tissue. Stem Cells 2006;24:1294-301.

[9] Kim J-H, Jo CH, Kim H-R, Hwang Y. Comparison of Immunological Characteristics of Mesenchymal Stem Cells from the Periodontal Ligament, Umbilical Cord, and Adipose Tissue. Stem Cells Int 2018;2018:1-12.

[10] Du WJ, Chi Y, Yang ZX, Li ZJ, Cui JJ, Song BQ et al. Heterogeneity of proangiogenic features in mesenchymal stem cells derived from bone marrow, adipose tissue, umbilical cord, and placenta. Stem Cell Res Ther 2016;7:163.

[11] Hsiao ST-F, Asgari A, Lokmic Z, Sinclair R, Dusting GJ, Lim SY, et al. Comparative Analysis of Paracrine Factor Expression in Human Adult Mesenchymal Stem Cells Derived from Bone Marrow, Adipose, and Dermal Tissue. Stem Cells Dev 2012;21:2189-203.

[12] Mattar P, Bieback K. Comparing the Immunomodulatory Properties of Bone Marrow, Adipose Tissue, and Birth-Associated Tissue Mesenchymal Stromal Cells. Front Immunol 2015;6:560.

[13] Paliwal S, Chaudhuri R, Agrawal A, Mohanty S. Human tissue-specific MSCs demonstrate differential mitochondria transfer abilities that may determine their regenerative abilities. Stem Cell Res Ther 2018;9:298.

[14] Lukomska B, Stanaszek L, Zuba-Surma E, Legosz P, Sarzynska S, Drela K. Challenges and Controversies in Human Mesenchymal Stem Cell Therapy. Stem Cells Int 2019;2019:1-10.

[15] Wagner W, Wein F, Seckinger A, Frankhauser M, Wirkner U, Krause U, et al. Comparative characteristics of mesenchymal stem cells from human bone marrow, adipose tissue, and umbilical cord blood. Exp Hematol 2005;33:1402-16.

[16] Vanda S L, Ngo A, Tzu Ni H. A Xeno-Free, Serum-Free Expansion Medium for Exvivo Expansion and Maintenance of Major Human Tissue-Derived Mesenchymal Stromal Cells. Transl Biomed 2018: 09.

[17] Phinney DG, Galipeau J. Manufacturing mesenchymal stromal cells for clinical applications: a survey of Good Manufacturing Practices at U.S. academic centers. Cytotherapy 2019;21:782-92.

[18] Dominici M, Le Blanc K, Mueller I, Slaper-Cortenbach I, Marini FC, Krause DS, et al. Minimal criteria for defining multipotent mesenchymal stromal cells. The International Society for Cellular Therapy position statement. Cytotherapy 2006;8:315-7.
[19] Esmaeli A, Moshrefi M, Shamsara A, Eftekhar-Vaghefi SH, Nematollahi-Mahani SN. Xeno-free culture condition for human bone marrow and umbilical cord matrix-derived mesenchymal stem/stromal cells using human umbilical cord blood serum. Int J Reprod Biomed Yazd Iran 2016;14:567-76.

[20] Heo JS, Choi Y, Kim H-S, Kim HO. Comparison of molecular profiles of human mesenchymal stem cells derived from bone marrow, umbilical cord blood, placenta and adipose tissue. Int J Mol Med 2016;37:115-25.

[21] Oikonomopoulos A, van Deen WK, Manansala A-R, Lacey PN, Tomakili TA, Ziman A, et al. Optimization of human mesenchymal stem cell manufacturing: the effects of animal/xeno-free media. Sci Rep 2015;5:16570.

[22] Cimino M, Gonçalves RM, Barrias CC, Martins MCL. Xeno-Free Strategies for Safe Human Mesenchymal Stem/Stromal Cell Expansion: Supplements and Coatings. Stem Cells Int 2017:2017:1-13.

[23] Chase LG, Yang S, Zachar V, Yang Z, Lakshmipathy U, Bradford J, et al. Development and Characterization of a Clinically Compliant Xeno-Free Culture Medium in Good Manufacturing Practice for Human Multipotent Mesenchymal Stem Cells. STEM CELLS Transl Med 2012;1:750-8.

[24] Swamynathan P, Venugopal P, Kannan S, Thej C, Kolkundar U, Bhagwat S, et al. Are serum-free and xeno-free culture conditions ideal for large scale clinical grade expansion of Wharton's jelly derived mesenchymal stem cells? A comparative study. Stem Cell Res Ther 2014;5:88.

[25] Tangjit N, Dechkunakorn S, Anuwongnukroh N, Khaneungthong A, Sritanaudomchai H. Optimal Xeno-free Culture Condition for Clinical Grade Stem Cells from Human Exfoliated Deciduous Teeth. Int J Stem Cells 2018;11:96-104.

[26] Wang Y, Wu H, Yang Z, Chi Y, Meng L, Mao A, et al. Human mesenchymal stem cells possess different biological characteristics but do not change their therapeutic potential when cultured in serum free medium. Stem Cell Res Ther 2014:5:132.

[27] Huang L, Su H. Developing a human mesenchymal stem cell culture medium with high growth efficient, 3d culture supported, serum-free and xeno-free properties. Cytotherapy 2019;21:e14

[28] Selich A, Daudert J, Hass R, Philipp F, von Kaisenberg C, Paul G, et al. Massive Clonal Selection and Transiently Contributing Clones During Expansion of Mesenchymal Stem Cell Cultures Revealed by Lentiviral RGB-Barcode Technology: Clonal Selection During Expansion of MSC Cultures. STEM CELLS Transl Med 2016;5:591-601.

[29] Mendicino M, Bailey AM, Wonnacott K, Puri RK, Bauer SR. MSC-Based Product Characterization for Clinical Trials: An FDA Perspective. Cell Stem Cell 2014;14:141-5.

[30] Office of Medical Products and Tobacco. Center for Devices and Radiological Health, Office of Medical Products and Tobacco, Center for Biologics Evaluation and Research. Regulatory Considerations for Human Cells, Tissues, and Cellular and Tissue-Based Products: Minimal Manipulation and Homologous Use Guidance for Industry and Food and Drug Administration Staff 2017.

[31] Alt EU, Senst C, Murthy SN, Slakey DP, Dupin CL, Chaffin AE, et al. Aging alters tissue resident mesenchymal stem cell properties. Stem Cell Res 2012;8:215-25.

[32] Marędziak M, Marycz K, Tomaszewski KA, Kornicka K, Henry BM. The Influence of Aging on the Regenerative Potential of Human Adipose Derived Mesenchymal Stem Cells. Stem Cells Int 2016:2016:1-15.

[33] Yang Y-HK. Aging of mesenchymal stem cells: implication in regenerative medicine. Regen Ther 2018;9:120-2.

[34] Ho Y-T, Shimbo T, Wijaya E, Ouchi Y, Takaki E, Yamamoto R, et al. Chromatin accessibility identifies diversity in mesenchymal stem cells from different tissue origins. Sci Rep 2018;8:17765.

[35] Li C, Wu X, Tong J, Yang X, Zhao J, Zheng Q et al. Comparative analysis of human mesenchymal stem cells from bone marrow and adipose tissue under xeno-free conditions for cell therapy. Stem Cell Res Ther 2015;6:55.

[36] Melief SM, Zwaginga JJ, Fibbe WE, Roelofs H. Adipose Tissue-Derived Multipotent Stromal Cells Have a Higher Immunomodulatory Capacity Than Their Bone Marrow-Derived Counterparts. STEM CELLS Transl Med 2013;2:455-63.

[37] Galipeau J, Sensébé L. Mesenchymal Stromal Cells: Clinical Challenges and Therapeutic Opportunities. Cell Stem Cell 2018;22:824-33.

[38] Hoogduijn MJ, Lombardo E. Mesenchymal Stromal Cells Anno 2019: Dawn of the Therapeutic Era? Concise Review. STEM CELLS Transl Med 2019;8:112634.

[39] MSC-Brew GMP Medium. Available at https://www.miltenyibiotec.com/CA-en/ products/cell-manufacturing-platform/macs-gmp-portfolio/cell-culture-media/ msc-brew-gmp-medium.html. Accessed April 23, 2020. 